\title{
Coronary spasm: unpredictability and safety in treatment
}

\author{
Carlotta Brega ${ }^{1}$, Eliana Raviola ${ }^{1}$, Emmanuel Lansac ${ }^{2}$, Fabio Zucchetta ${ }^{1}$, Alberto Tripodi $^{1}$, \\ and Alberto Albertini ${ }^{3}$ \\ ${ }^{1}$ Maria Cecilia Hospital, GVM care and research \\ ${ }^{2}$ Institut Mutualiste Montsouris \\ ${ }^{3}$ Maria Cecilia Hospital SpA
}

September 10, 2020

\begin{abstract}
The present case describes the severity of postoperative coronary spasm after aortic valve sparing surgery. It highlights the crucial role of hybrid setting for diagnosis and treatment of the disease.
\end{abstract}

\section{Case report}

We report the clinical case of a young 50-year-old woman, affected by severe regurgitation of bicuspid aortic valve associated to aortic aneurysm. In the past she had radio and chemotherapy for cancer of the right breast. She was symptomatic for dyspnea for moderate effort (NYHA II) and she had no other cardiac symptom. Transesophageal echocardiography (TEE) showed normal ejection fraction (EF), normal left ventricle diameters and a severely incontinent type I bicuspid aortic valve: the rafe was between right and left coronary cusp and the non-coronary cusp was prolapsing (Figure 1). A coronary artery CT scan angiography reported the absence of coronary stenosis and aortic dilation $(49.6 \mathrm{~mm}$ as aortic root diameter and $47 \mathrm{~mm}$ diameter of the ascending aorta) (Figure 2). According to the current guidelines ${ }^{1}$, surgical indication for aortic valve repair and root- ascending aorta replacement was posed. The operation was performed though median sternotomy and cardiopulmonary by-pass $(\mathrm{CPB})$ was established through right atrium and distal aortic arch cannulation. Cristalloid cold cardioplegia (Custodiol) was administered directly in the coronary ostia. No anomalies in the origin of the coronary arteries was found and the ostia were normally positioned. Operative inspection confirmed the echocardiographic data: aortic valve was bicuspid, with a coronary cusp resulting from the fusion of the right and the left coronary cusp and a normal non-coronary cusp. Aortic annulus was dilated and measured with Hegar dilator at $28 \mathrm{~mm}$. Aortic valve repair was performed through the excision of the rafe on the coronary cusp; then, plicating stitches on the free edge were put, in order to realign the free edges, aiming for an effective height of $9 \mathrm{~mm}^{2}$. Remodelling of the aortic root with subvalvular external annuloplasty (Cardioroot bulged graft $28 \mathrm{~mm}$ and CORONEOß ring $27 \mathrm{~mm}$ ) was performed ${ }^{2}$. Weaning from CBP was uneventful and TEE showed a normal functional valve with no insufficiency and no pathological gradients. After weaning, hemodynamical instability suddenly developed: episodes of ventricular fibrillation (FV) were repeatedly shocked and ST elevation was reported in the inferior leads. Hemodynamic deterioration was not improved by intra-aortic balloon pump (IABP) and CBP was restarted. A venous graft was used to bypass the right coronary artery, but unsuccessfully. As the operative theatre was hybrid, coronary angiogram was logistically easy and it was promptly performed. Angiography showed massive coronary spasm (Figure 3), not responsive to intracoronary nitrates, so balloon angioplasty and drug eluted stents were placed in the right, circumflex and anterior descending coronary arteries (Figure 4). CPB weaning was then possible with inotropic support and the patient was moved to the ICU. During the first postoperative day the maximum troponin release was $2732 \mathrm{ng} / \mathrm{l}$ (14-50 cut off). Hemodynamical conditions gradually improved and in second postoperative day IABP removal and extubation were possible. 
Inotropic drug support was step by step reduced e definitely stopped at the $72^{\text {nd }}$ postoperative hour. In fourth postoperative day the patient was moved to the ward and discharged home in a few days. The echocardiography before discharge showed no incompetence, no pathological gradients on the aortic valve and a normal ejection fraction (Figure 5), a coronary artery CT scan angiography showed excellent result with no residual coronary artery stenoses (Figure 6). At follow-up outpatient visits, at one and six months, the patient was fine and asymptomatical.

\section{Discussion}

Coronary spasm after cardiac surgery is a rare entity that can be associated to the development of arrythmias, both ventricular fibrillation or ventricular tachycardia and bradycardia and atrioventricular blocks ${ }^{3}$. It is defined as transient total or subtotal coronary artery occlusion with angina and ischemic ECG changes ${ }^{4}$. The underlying mechanism in this process is still not clear and it results from the accumulation of multiple triggers including endothelial dysfunction, coronary smooth muscle calcium hypersensitivity, magnesium insufficiency, CBP related inflammation and genetic susceptibility ${ }^{5}$. Recently, coronary arteries hypersensitivity with vasospasm has been defined as Kounis Syndrome ${ }^{6}$ : anaphylactic response is supposed to be the origin of a spectrum of coronary diseases resulting in microvascular endothelial dysfunction and coronary spasm. Diagnosis may be challenging without invasive examination, so certitude is obtained only after coronary angiography. Anyway, coronary spasm should be suspected in case of electrical instability after cardiac surgery: it occurs especially after surgical myocardial revascularization, rarely after valvular surgery, as reported in literature. Thus this syndrome is unpredictable, promptness in diagnosis and treatment is fundamental. Early recognition and immediate institution of specific treatment can be performed in a hybrid theatre ${ }^{7}$ : open-chest coronary angiography allows evaluation and treatment of patients with early cardiovascular collapse after cardiac surgery. Furthermore, the consequent cardiac events such as coronary artery disease and acute myocardial ischemia play crucial role in patient survival ${ }^{8}$. Angioplasty (PCI) and coronary stenting are not routinely indicated for patients with focal spasm and minimal artery disease. Spasms may be due to the withdrawal of long-acting oral nitrates or calcium channel blockers and are usually treated with intracoronary nitrates, but it was not our case, for both, as the patient had no therapy preoperatively and coronary spasms did not respond to vasodilators nor to IABP. Moreover, when spasm is refractory to conventional vasodilators, including nitrates, calcium channel blockers, PCI turns out to be a valuable option ${ }^{9}$. Stent placement may be effective and moreover life-saving, once hemodynamical instability has developed. Even if stent placement may be a risk, as it may induce itself end-stent spasms ${ }^{10}$, in our case, the decision of treatment was forced by the emergency situation, as our patient could not be weaned from CBP. The binominal interventional therapy and the opportunity to work in a hybrid setting have a key role in the management and in the clinical consequences.

[1] AlecVahanian, OttavioAlfieri, Felicita Andreotti, Manuel J. Antunes, Gonzalo Baro 'n-Esquivias, et al Guidelines on the management of valvular heart disease European Heart Journal (2012) 33, 2451-2496 doi:10.1093/eurheartj/ehs109

[2] Youssefi P, Zacek P, Debauchez M, Lansac E.Valve-Sparing Aortic Root Replacement Using the Remodeling Technique With Aortic Annuloplasty: Bicuspid Valves With Repair of Specific Lesion Sets: How I Teach It. Ann Thorac Surg. 2019 Aug;108(2):324-333. doi: 10.1016/j.athoracsur.2019.05.011. Epub 2019 Jun 15.

[3] H. Kishida, Y. Tada, N. Fukuma, et al., Significant characteristics of variant angina patients with associated syncope, Jpn. Heart J. 37 (1996) 317-326.

[4] BeltrameJF, CreaF, KaskiJC, OgawaH, OngP, SechtemU, et al., On Behalf of the Coronary Vasomotion Disorders International Study Group (COVADIS): International Standardization of diagnostic criteria for vasospastic angina. Eur. Heart J. http://dx. doi.org/10.1093/eurheartj/ehv351.

[5]G.A. Lanza, G. Careri, F. Crea, Contemporary reviews in cardiovascular medicine: mechanisms of coronary artery spasm, Circulation $124 \quad$ (2011) 1774-1782, http:// dx.doi.org/10.1161/CIRCULATIONAHA.111.037283. 
[6] Nicholas G. Kounis, Kounis syndrome: an update on epidemiology, pathogenesis, diagnosis and therapeutic

Management Clin Chem Lab Med 2016; 54(10): 1545-1559

[7] Skarvan K, Graedel E, Hasse J, Stulz P, Pfisterer M. Coronary artery spasms after coronary artery bypass surgery. Anesthesiology. 1984;61:323-7.

[8] A. Walling, D.D. Waters, D.D. Miller, D. Roy, G.B. Pelletier, P. Theroux, Long-term prognosis of patients with variant angina, Circulation 76 (5) (1987) 990-997.

[9] Yazdchi S, Desai S, Rodriguez J, et al. (February 18, 2020) Refractory Right Coronary Artery Spasm: A Case Report. Cureus 12(2): e7034. DOI 10.7759/cureus.7034

[10] Kaku B, Honin IK, Horita Y, et al. The incidence of stent-edge spasm after stent implantation in patients with or without vasospastic angina pectoris. Int Heart J 2005; 46: 23-33.

\section{Figures}

Figure 1. TEE images: aortic valve insufficiency with severe eccentric jet, towards anterior mitral leaflet; prolapse of the non-coronary cusp.

Figure 2. CT: aortic root measurement $49.6 \mathrm{~mm}$; ascending aorta $47 \mathrm{~mm}$ diameter; $32 \mathrm{~mm}$ diameter aortic arch. Absence of critical coronary stenosis.

Figure 3. Postoperative coronary angiography showing critical lesions on the posterior interventricular artery, on the circumflex artery, on the left descending anterior, on the diagonal branch

Figure 4. Coronary angiography after stent placement on the three coronary arteries. Hemodynamical stabilization with inotropes and transfer to the ICU

Figure 5. Postoperative transthoracic echocardiography showing no residual aortic insufficiency and good contractile function

Figure 6. Postoperative CT scan, showing vascular prosthesis and venous graft on the right coronary artery.

\section{Supplementary files}

Video 1. TEE PRE: Preoperative TEE showing severe aortic valve incompetence and non-coronary cusp prolapse in type1 bicuspid aortic valve.

Video 2. In-theatre TEE: no residual aortic valve insufficiency after repair.

Video 3. Postoperative coronary angiography: ight coronary angiography showing functioning venous graft and diffuse coronary spasm; severe spasm on the left descending anterior coronary artery (LAD) and on the circumflex coronary artery $(\mathrm{CX})$; stenting of the right coronary artery; stenting on the medium LAD; stenting on the ostial CX; final result of the PCI on the right coronary artery; final result of the PCI on the left coronary artery. 

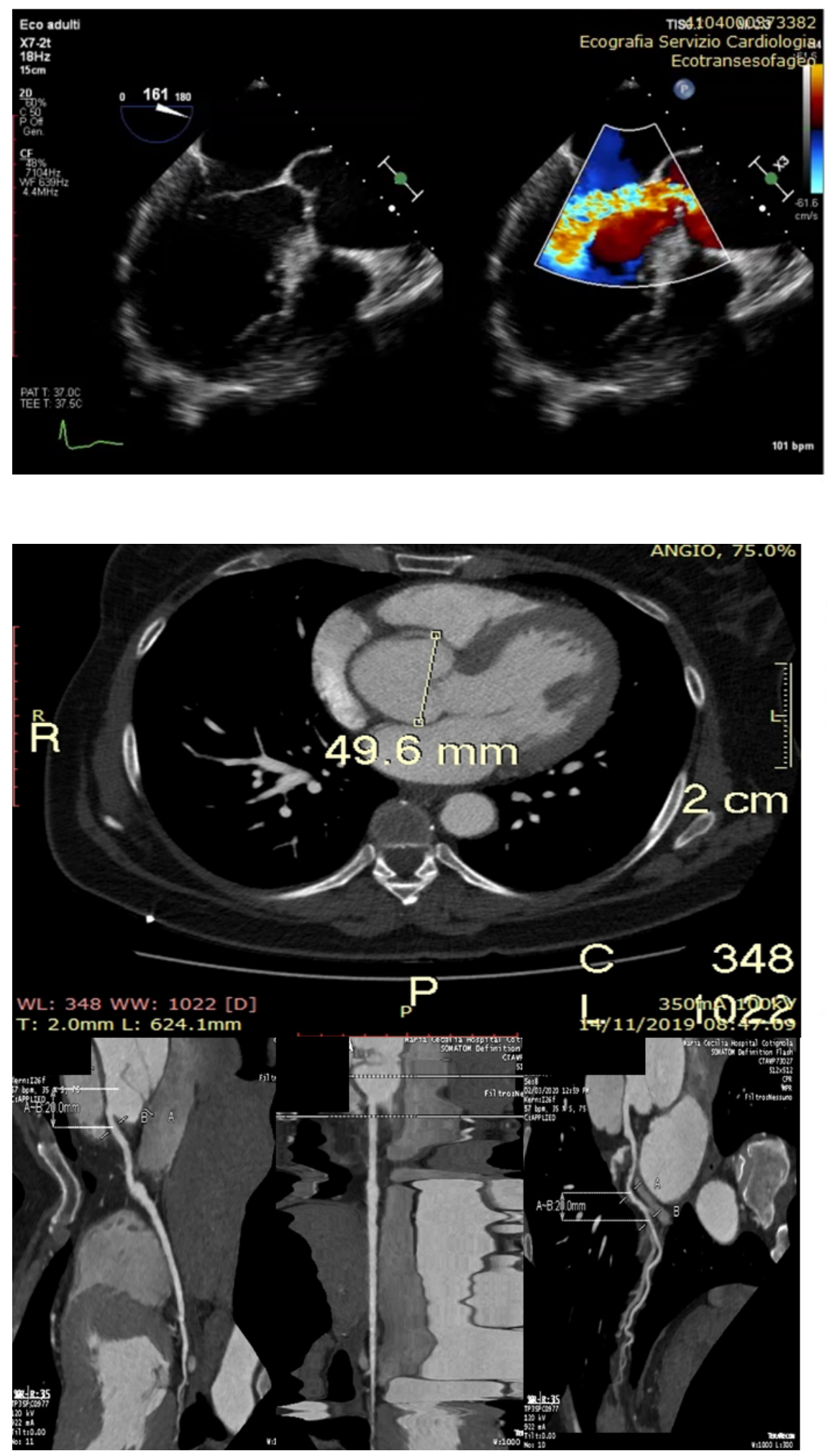

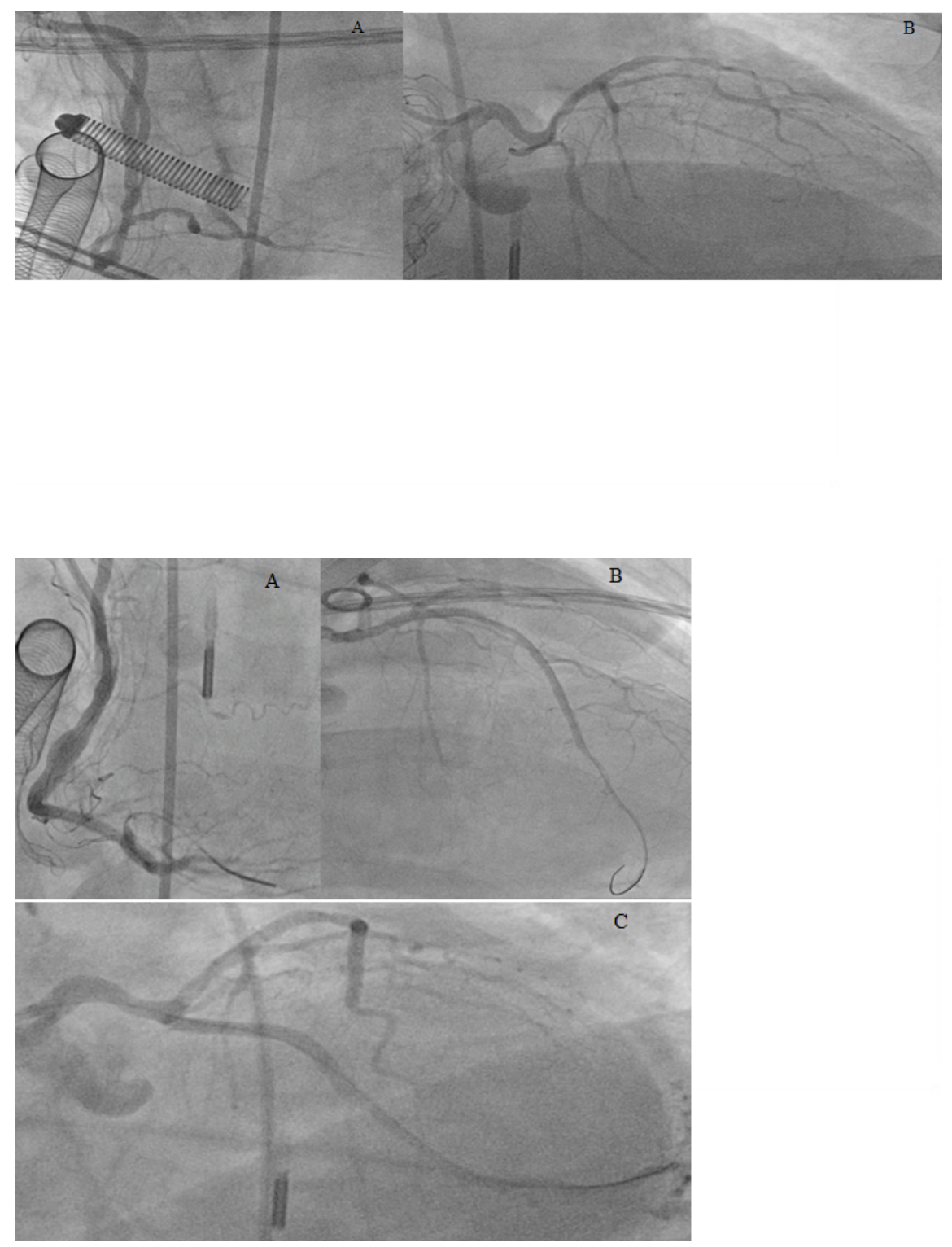

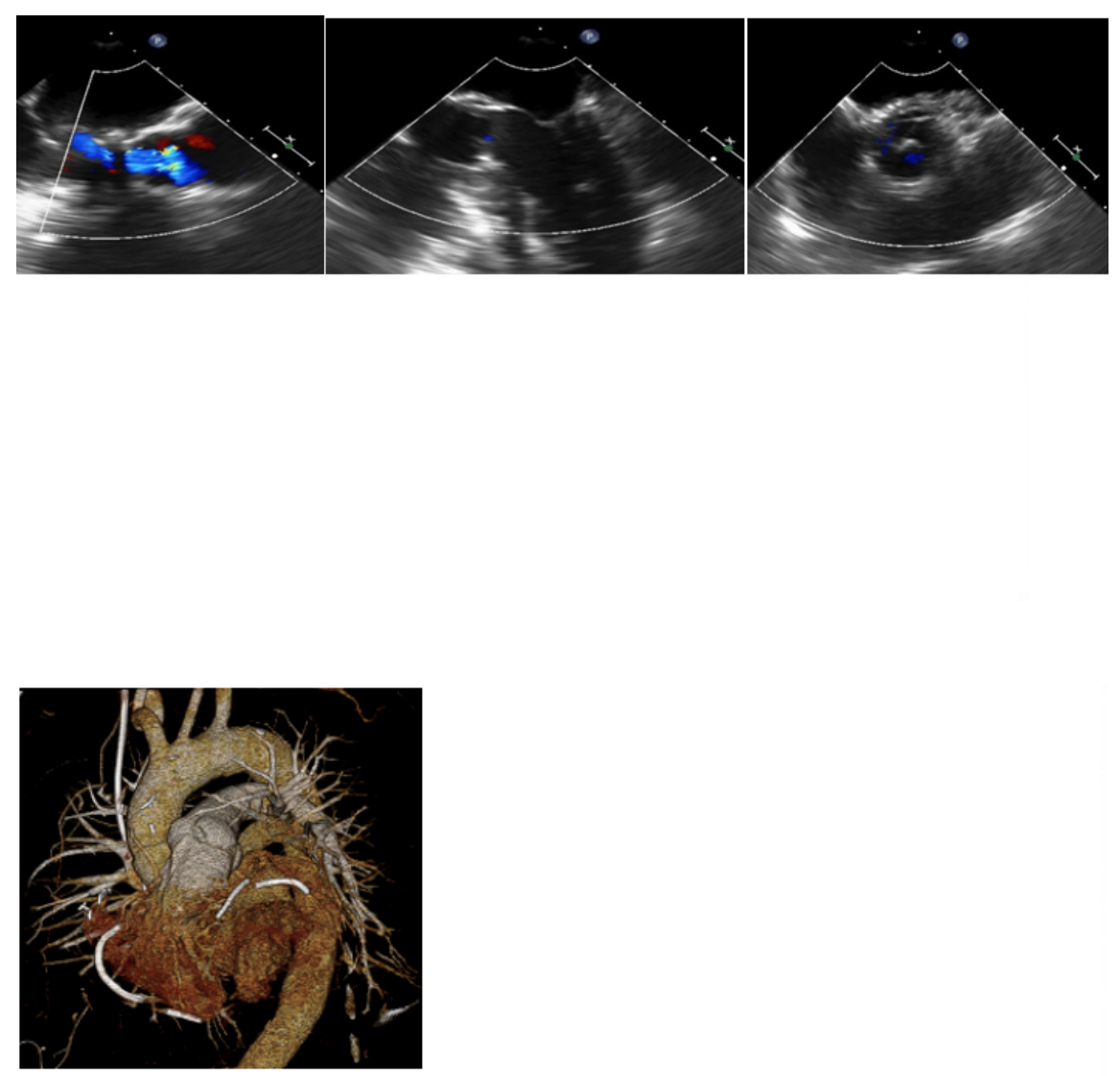